\title{
Dynamical Systems Revisited: Hybrid Systems with Zeno Executions ${ }^{\star}$
}

\author{
Jun Zhang, Karl Henrik Johansson`^, John Lygeros, and Shankar Sastry \\ Department of Electrical Engineering and Computer Sciences \\ University of California, Berkeley, CA 94720-1770 \\ \{zhangjun, johans, lygeros, sastry\}@eecs . berkeley.edu \\ http://www.eecs.berkeley.edu/ $\{$ zhangjun, johans, lygeros, sastry\}
}

\begin{abstract}
Results from classical dynamical systems are generalized to hybrid dynamical systems. The concept of $\omega$ limit set is introduced for hybrid systems and is used to prove new results on invariant sets and stability, where Zeno and non-Zeno hybrid systems can be treated within the same framework. As an example, LaSalle's Invariance Principle is extended to hybrid systems. Zeno hybrid systems are discussed in detail. The $\omega$ limit set of a Zeno execution is characterized for classes of hybrid systems.
\end{abstract}

\section{Introduction}

Systems with interacting continuous-time and discrete-time dynamics are used as models in a large variety of applications. The rich structure of such hybrid systems allow them to accurately predict the behavior of quite complex systems. However, the continuous-discrete nature of the system calls for new system theoretical tools for modeling, analysis, and design. Intensive recent activity have provided a few such tools, for instance, Lyapunov stability results [114. However, as will be shown in this paper, in many cases the results come with assumptions that are not only hard to check but also unnecessary. There are several fundamental properties of hybrid systems that have not been sufficiently studied in the literature. These include questions on existence and uniqueness of executions, which have only recently been addressed [127]. Another question is when a hybrid system exhibits an infinite number of discrete transitions during a finite time interval, which is referred to as Zeno. The significance of these questions has been pointed out by many researchers, e.g., He and Lemmon [3]

\footnotetext{
* This work was supported by ARO under the MURI grant DAAH04-96-1-0341, the Swedish Foundation for International Cooperation in Research and Higher Education, Telefonaktiebolaget LM Ericsson's Foundation, ONR under grant N00014-971-0946, and DARPA under contract F33615-98-C-3614.

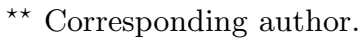


write "An important issue [ . . ] concerns necessary and sufficient conditions for a switched system to be live, deadlock free, or nonZeno."

The main contribution of the paper is to carefully generalize concepts from classical dynamical systems like $\omega$ limit sets and invariant sets, in a way so that Zeno executions are treated within the same framework as regular non-Zeno executions. It is then straightforward to extend existing results, for instance, Lyapunov stability theorems for hybrid systems [114. We illustrate this by proving LaSalle's Invariance Principle for hybrid systems.

Zeno is an interesting mathematical property of some hybrid systems, which does not occur in smooth dynamical systems. Real physical systems are not Zeno. Models of physical systems may, however, be Zeno due to a too high level of abstraction. In the latter part of the paper, we characterize Zeno executions and their Zeno states, where the Zeno states are defined as the $\omega$ limit points of a Zeno execution. We are able to completely characterize the set of Zeno states for a few classes of hybrid systems. It is shown that the features of the reset maps are important. For example, if the resets are identity maps or the resets are contractions, the continuous part of the Zeno state is a singleton.

The outline of the paper is as follows. In Section 2 notation and some basic definitions of hybrid automata and executions are introduced. Some recent results on existence and uniqueness of executions for classes of hybrid automata are also given. Section 3 introduces invariants sets and $\omega$ limit sets for hybrid automata and gives a generalization of LaSalle's Invariance Principle. Finally, results on Zeno hybrid automata are given in Section 4 , where for instance the $\omega$ limit set for Zeno executions are discussed and some necessary and sufficient conditions for Zenoness are given.

\section{Hybrid Automata and Executions}

\section{$2.1 \quad$ Notation}

For a finite collection $V$ of variables, let $\mathbf{V}$ denote the set of valuations of these variables. We use lower case letters to denote both a variable and its valuation. We refer to variables whose set of valuations is finite or countable as discrete and to variables whose set of valuations is a subset of a Euclidean space as continuous. For a set of continuous variables $X$ with $\mathbf{X}=\mathbb{R}^{n}$ for $n \geq 0$, we assume that $\mathbf{X}$ is given the Euclidean metric topology, and use $\|\cdot\|$ to denote the Euclidean norm. For a set of discrete variables $Q$, we assume that $\mathbf{Q}$ is given the discrete topology (every subset is an open set), generated by the metric $d_{D}\left(q, q^{\prime}\right)=0$ if $q=q^{\prime}$ and $d_{D}\left(q, q^{\prime}\right)=1$ if $q \neq q^{\prime}$. We denote the valuations of the union $Q \cup X$ by $\mathbf{Q} \times \mathbf{X}$, which is given the product topology, generated by the metric $d\left((q, x),\left(q^{\prime}, x^{\prime}\right)\right)=d_{D}\left(q, q^{\prime}\right)+\left\|x-x^{\prime}\right\|$. Using the metric $d$, we define the distance between two sets $U_{1}, U_{2} \subseteq \mathbf{Q} \times \mathbf{X}$ by $d\left(U_{1}, U_{2}\right)=\inf _{\left(q_{i}, x_{i}\right) \in U_{i}} d\left(\left(q_{1}, x_{1}\right),\left(q_{2}, x_{2}\right)\right)$. We assume that a subset $U$ of a topological space is given the induced topology, and we use $\bar{U}$ to denote its closure, $U^{o}$ its interior, $\partial U$ its boundary, $U^{c}$ its complement, $|U|$ its cardinality, and $P(U)$ the set of all subsets of $U$. 


\subsection{Basic Definitions}

The following definitions are based on 8,477.

Definition 1 (Hybrid Automaton). A hybrid automaton $H$ is a collection $H=(Q, X$, Init, $f$, Dom, Reset $)$, where

$-Q$ is a finite collection of discrete variables;

- $X$ is a finite collection of continuous variables with $\mathbf{X}=\mathbb{R}^{n}$;

- Init $\subseteq \mathbf{Q} \times \mathbf{X}$ is a set of initial states;

$-f: \mathbf{Q} \times \mathbf{X} \rightarrow T \mathbf{X}$ is a vector field;

- Dom $\subseteq \mathbf{Q} \times \mathbf{X}$ is the domain of $H, 1$

- Reset : $\mathbf{Q} \times \mathbf{X} \rightarrow P(\mathbf{Q} \times \mathbf{X})$ is a reset relation.

We refer to $(q, x) \in \mathbf{Q} \times \mathbf{X}$ as the state of $H$. Unless otherwise stated, we introduce the following assumption, to prevent some obvious pathological cases.

Assumption $1|\mathbf{Q}|<\infty$ and $f$ is Lipschitz continuous in its second argument.

Note that, under the discrete topology on $\mathbf{Q}, f$ is trivially continuous in its first argument. A hybrid automaton can be represented by a directed graph $(\mathbf{Q}, E)$, with vertices $\mathbf{Q}$ and edges

$$
E=\left\{\left(q, q^{\prime}\right) \in \mathbf{Q} \times \mathbf{Q}: \exists x, x^{\prime} \in \mathbf{X},\left(q^{\prime}, x^{\prime}\right) \in \operatorname{Reset}(q, x)\right\} .
$$

With each vertex $q \in \mathbf{Q}$, we associate a set of continuous initial states

$$
\operatorname{Init}(q)=\{x \in \mathbf{X}:(q, x) \in \operatorname{Init}\},
$$

a vector field $f(q, \cdot)$, and a set

$$
I(q)=\{x \in \mathbf{X}:(q, x) \in \operatorname{Dom}\} .
$$

With each edge $e=\left(q, q^{\prime}\right) \in E$, we associate a guard

$$
G(e)=\left\{x \in \mathbf{X}: \exists x^{\prime} \in \mathbf{X},\left(q^{\prime}, x^{\prime}\right) \in \operatorname{Reset}(q, x)\right\},
$$

and a reset map

$$
R(e, x)=\left\{x^{\prime} \in \mathbf{X}:\left(q^{\prime}, x^{\prime}\right) \in \operatorname{Reset}(q, x)\right\} .
$$

Since there is a unique graphical representation for each hybrid automaton, we will use the corresponding graphs as formal definitions for hybrid automata in most examples.

\footnotetext{
${ }^{1}$ The set Dom is often called the invariant set in the hybrid system literature in computer science. We reserve this term for later in the paper, where we will discuss sets invariant in the usual dynamical systems sense.
} 
Definition 2 (Hybrid Time Trajectory). A hybrid time trajectory $\tau$ is a finite or infinite sequence of intervals $\tau=\left\{I_{i}\right\}_{i=0}^{N}$, such that

$$
\begin{aligned}
& -I_{i}=\left[\tau_{i}, \tau_{i}^{\prime}\right] \text { for } i<N, \text { and, if } N<\infty, I_{N}=\left[\tau_{N}, \tau_{N}^{\prime}\right] \text { or } I_{N}=\left[\tau_{N}, \tau_{N}^{\prime}\right) \text {; and } \\
& -\tau_{i} \leq \tau_{i}^{\prime}=\tau_{i+1} \text { for } i \geq 0 .
\end{aligned}
$$

A hybrid time trajectory is a sequence of intervals of the real line, whose end points overlap. The interpretation is that the end points of the intervals are the times at which discrete transitions take place. Note that $\tau_{i}=\tau_{i}^{\prime}$ is allowed, therefore multiple discrete transitions may take place at the same "time". Since the dynamical systems we will be concerned with are time invariant we will sometimes, without loss of generality, assume $\tau_{0}=0$. Hybrid time trajectories can extend to infinity if $\tau$ is an infinite sequence or if it is a finite sequence ending with an interval of the form $\left[\tau_{N}, \infty\right)$. We denote by $\mathcal{T}$ the set of all hybrid time trajectories and use $t \in \tau$ as shorthand notation for that there exists $i$ such that $t \in I_{i} \in \tau$. For a topological space $K$ we use $k: \tau \rightarrow K$ as a short hand notation for a map assigning a value from $K$ to each $t \in \tau$; note that $k$ is not a function on the real line, as it assigns multiple values to the same $t \in \mathbb{R}: t=\tau_{i}^{\prime}=\tau_{i+1}$ for all $i \geq 0$. Each $\tau \in \mathcal{T}$ is fully ordered by the relation $\prec$ defined by $t_{1} \prec t_{2}$ for $t_{1} \in\left[\tau_{i}, \tau_{i}^{\prime}\right]$ and $t_{2} \in\left[\tau_{j}, \tau_{j}^{\prime}\right]$ if and only if $i<j$, or $i=j$ and $t_{1}<t_{2}$.

Definition 3 (Execution). An execution $\chi$ of a hybrid automaton $H$ is a collection $\chi=(\tau, q, x)$ with $\tau \in \mathcal{T}, q: \tau \rightarrow \mathbf{Q}$, and $x: \tau \rightarrow \mathbf{X}$, satisfying

$-\left(q\left(\tau_{0}\right), x\left(\tau_{0}\right)\right) \in$ Init (initial condition);

- for all $i$ with $\tau_{i}<\tau_{i}^{\prime}, q(\cdot)$ is constant and $x(\cdot)$ is a solution to the differential equation $d x / d t=f(q, x)$ over $\left[\tau_{i}, \tau_{i}^{\prime}\right]$, and for all $t \in\left[\tau_{i}, \tau_{i}^{\prime}\right),(q(t), x(t)) \in$ Dom (continuous evolution); and

- for all $i,\left(q\left(\tau_{i+1}\right), x\left(\tau_{i+1}\right)\right) \in \operatorname{Reset}\left(q\left(\tau_{i}^{\prime}\right), x\left(\tau_{i}^{\prime}\right)\right)$ (discrete evolution).

We say a hybrid automaton accepts an execution $\chi$ or not. For an execution $\chi=(\tau, q, x)$, we use $\left(q_{0}, x_{0}\right)=\left(q\left(\tau_{0}\right), x\left(\tau_{0}\right)\right)$ to denote the initial state of $\chi$. The execution time $\tau_{\infty}(\chi)$ is defined as $\tau_{\infty}(\chi)=\sum_{i=0}^{N}\left(\tau_{i}^{\prime}-\tau_{i}\right)$, where $N+1$ is the number of intervals in the hybrid time trajectory. The argument $\chi$ will sometimes be left out. An execution is finite if $\tau$ is a finite sequence ending with a compact interval, it is called infinite if $\tau$ is either an infinite sequence or if $\tau_{\infty}(\chi)=\infty$, and it is called Zeno if it is infinite but $\tau_{\infty}(\chi)<\infty$. The execution time of a Zeno execution is also called the Zeno time. We use $\mathcal{E}_{H}\left(q_{0}, x_{0}\right)$ to denote the set of all executions of $H$ with initial condition $\left(q_{0}, x_{0}\right) \in \operatorname{Init}, \mathcal{E}_{H}^{\infty}\left(q_{0}, x_{0}\right)$ to denote the set of all infinite executions of $H$ with initial condition $\left(q_{0}, x_{0}\right) \in$ Init. We define $\mathcal{E}_{H}=\bigcup_{\left(q_{0}, x_{0}\right) \in \operatorname{Init}} \mathcal{E}_{H}\left(q_{0}, x_{0}\right)$ and $\mathcal{E}_{H}^{\infty}=\bigcup_{\left(q_{0}, x_{0}\right) \in \operatorname{Init}} \mathcal{E}_{H}^{\infty}\left(q_{0}, x_{0}\right)$. To simplify the notation, we will drop the subscript $H$ whenever the automaton is clear from the context.

$\overline{2}$ "Solution" is interpreted in the sense of Caratheodory. 


\subsection{Classes of Automata}

The notation previously introduced gives a convenient way to express existence and uniqueness of executions.

Definition 4 (Non-Blocking Automaton). A hybrid automaton $H$ is nonblocking if $\mathcal{E}_{H}^{\infty}\left(q_{0}, x_{0}\right)$ is non-empty for all $\left(q_{0}, x_{0}\right) \in$ Init.

Definition 5 (Deterministic Automaton). A hybrid automaton $H$ is deterministic if $\mathcal{E}_{H}^{\infty}\left(q_{0}, x_{0}\right)$ contains at most one element for all $\left(q_{0}, x_{0}\right) \in$ Init.

Note that if a hybrid automaton is both non-blocking and deterministic, then it accepts a unique infinite execution for each initial condition. In [7] conditions were established that determine whether an automaton is non-blocking and deterministic. The conditions require one to argue about the set of states reachable by a hybrid automaton, and the set of states from which continuous evolution is impossible. A state $(q, x) \in \mathbf{Q} \times \mathbf{X}$ is called reachable by $H$, if there exists a finite execution $\chi=(\tau, q, x)$ with $\tau=\left\{\left[\tau_{i}, \tau_{i}^{\prime}\right]\right\}_{i=0}^{N}$ and $\left(q\left(\tau_{N}^{\prime}\right), x\left(\tau_{N}^{\prime}\right)\right)=(q, x)$. We use $\operatorname{Reach}_{H}$ to denote the set of states reachable by a hybrid automaton, and $\operatorname{Reach}_{H}(q)$ the projection of $\operatorname{Reach}_{H}$ to discrete state $q$. We will drop the subscript $H$ whenever the automaton is clear from the context. The set Reach is in general difficult to compute. Fortunately, the conditions of the subsequent results will not require us to do so: any outer approximation of the reachable set will be sufficient. In [2]7 methods for computing such outer approximations using simple induction arguments are outlined.

The set of states from which continuous evolution is impossible is given by

$$
\mathrm{Out}_{H}=\left\{\left(q^{0}, x^{0}\right) \in \mathbf{Q} \times \mathbf{X}: \forall \epsilon>0, \exists t \in[0, \epsilon),\left(q^{0}, x(t)\right) \notin \operatorname{Dom}\right\},
$$

where $x(\cdot)$ is the solution to $d x / d t=f\left(q^{0}, x\right)$ with $x(0)=x^{0}$. Note that if Dom is an open set, then Out is simply Dom ${ }^{c}$. If Dom is closed, then Out may also contain parts of the boundary of Dom. In [7] methods for computing Out were proposed, under appropriate smoothness assumptions on $f$ and the boundary of Dom. As before, we will use $\operatorname{Out}_{H}(q)$ to denote the projection of Out to discrete state $q$, and drop the subscript $H$ whenever the automaton is clear from the context. With these two pieces of notation one can show the following two results [7].

Proposition 1. A (deterministic) hybrid automaton is non-blocking if (and only if) for all $(q, x) \in$ Out $\cap \operatorname{Reach}$, $\operatorname{Reset}(q, x) \neq \emptyset$.

Proposition 2. A hybrid automaton is deterministic if and only if for all $(q, x) \in \operatorname{Reach},|\operatorname{Reset}(q, x)| \leq 1$ and, if $\operatorname{Reset}(q, x) \neq \emptyset,(q, x) \in$ Out.

We characterize the hybrid automata such that the state remains in the closure of the invariant along all executions.

Definition 6 (Domain Preserving). A hybrid automaton is domain preserving if Reach $\subseteq \overline{\mathrm{Dom}}$. 
The following result is now straightforward.

Proposition 3. A hybrid automaton is domain preserving if and only if Init $\subseteq$ Dom and for all $(q, x) \in \operatorname{Dom} \cap \operatorname{Reach}, \operatorname{Reset}(q, x) \subseteq \operatorname{Dom}$.

Note that the use of Reach is again not limiting. Note also that the conditions of the lemma do not depend on the vector field $f$. This is because, by the definition of an execution, the state can never end up outside the closure of the domain along continuous evolution.

Definition 7 (Transverse Domain). A hybrid automaton $H$ is said to have transverse domain if there exists a function $\sigma: \mathbf{Q} \times \mathbf{X} \rightarrow \mathbb{R}$ continuously differentiable in its second argument, such that

$$
\operatorname{Dom}=\{(q, x) \in \mathbf{Q} \times \mathbf{X}: \sigma(q, x) \geq 0\}
$$

and for all $(q, x)$ with $\sigma(q, x)=0, L_{f} \sigma(q, x) \neq 0$.

Here $L_{f} \sigma: \mathbf{Q} \times \mathbf{X} \rightarrow \mathbb{R}$ denotes the Lie derivative of $\sigma$ along $f$ defined as

$$
L_{f} \sigma(q, x)=\frac{\partial \sigma}{\partial x}(q, x) \cdot f(q, x)
$$

In other words, an automaton has transverse domain if the set Dom is closed, its boundary is differentiable, and the vector field $f$ is pointing either inside or outside of Dom along the boundary 3 If $H$ has transverse domain the set $\mathrm{Out}_{H}$ admits a fairly simple characterization.

Proposition 4. If $H$ has transverse domain, then

$$
\begin{aligned}
\text { Out }_{H}=\{( & (q, x) \in \mathbf{Q} \times \mathbf{X}: \sigma(q, x)<0\} \\
& \cup\left\{(q, x) \in \mathbf{Q} \times \mathbf{X}: \sigma(q, x)=0 \text { and } L_{f} \sigma(q, x)<0\right\} .
\end{aligned}
$$

\section{Invariant Sets and Stability}

We first recall some standard concepts from dynamical system theory, and discuss how they generalize to hybrid automata.

Definition 8 (Invariant Set). A set $M \subseteq$ Init is called invariant if for all $\left(q_{0}, x_{0}\right) \in M,(\tau, q, x) \in \mathcal{E}_{H}\left(q_{0}, x_{0}\right)$, and $t \in \tau$, it holds that $(q(t), x(t)) \in M$.

The class of invariant sets is closed under arbitrary unions and intersections. Invariant sets are such that all executions starting in the set remain in the set for ever. We are interested in studying the stability of invariant sets, i.e., determine whether all trajectories that start close to an invariant set remain close to it.

\footnotetext{
${ }^{3}$ Under appropriate smoothness assumptions on $\sigma$ and $f$ the definition of transverse domain can be relaxed somewhat by allowing $L_{f} \sigma(q, x)=0$ on the boundary of Dom and taking higher-order Lie derivatives, until one that is non-zero is found. Even though many of the results presented here extend to this relaxed definition, the proofs are slightly more technical. We will therefore limit ourselves to the notion of transverse domain given in Definition 7
} 
Definition 9 (Stable Invariant Set). An invariant set $M \subseteq$ Init is called stable if for all $\epsilon>0$ there exists $\delta>0$ such that for all $\left(q_{0}, x_{0}\right) \in$ Init, with $d\left(\left(q_{0}, x_{0}\right), M\right)<\delta$, all $(\tau, q, x) \in \mathcal{E}_{H}\left(q_{0}, x_{0}\right)$, and all $t \in \tau, d((q(t), x(t)), M)<\epsilon$.

An invariant set is called (locally) asymptotically stable if it is stable and in addition there exists $\Delta>0$ such that for all $\left(q_{0}, x_{0}\right) \in \operatorname{Init}$, with $d\left(\left(q_{0}, x_{0}\right), M\right)<$ $\Delta$, and all $(\tau, q, x) \in \mathcal{E}_{H}^{\infty}\left(q_{0}, x_{0}\right), \lim _{t \rightarrow \tau_{\infty}} d((q(t), x(t)), M)=0$.

Note that since $\tau$ is fully ordered the above limit is well defined. The asymptotic behavior of an infinite execution is captured in terms of its $\omega$ limit set.

Definition 10 ( $\omega$ limit set). The $\omega$ limit point $(\hat{q}, \hat{x}) \in \mathbf{Q} \times \mathbf{X}$ of an execution $\chi=(\tau, q, x) \in \mathcal{E}_{H}^{\infty}$ is a point for which there exists a sequence $\left\{\theta_{n}\right\}_{n=0}^{\infty}, \theta_{n} \in \tau$, such that as $n \rightarrow \infty, \theta_{n} \rightarrow \tau_{\infty}$ and $\left(q\left(\theta_{n}\right), x\left(\theta_{n}\right)\right) \rightarrow(\hat{q}, \hat{x})$. The $\omega$ limit set $S_{\chi} \subseteq \mathbf{Q} \times \mathbf{X}$ is the set of all $\omega$ limit points of an execution $\chi$.

The following lemma establishes a relation between $\omega$ limit sets and invariant sets. For convenience the assumptions on the reset relation and the domain are given in the graphical notation introduced in Section 2.2

Lemma 1. Consider a deterministic hybrid automaton $H$ with transverse domain. Assume it is domain preserving and that $f(q, \cdot)$ is $C^{1}$ for all $q \in \mathbf{Q}$. Furthermore, assume that for all $e=\left(q, q^{\prime}\right) \in E, R(e, \cdot)$ is continuous, and $G(e) \cap I(q)$ is an open subset of $\partial I(q)$. Then, for any execution $\chi=(\tau, q, x) \in$ $\mathcal{E}_{H}^{\infty}$, if $x(\cdot)$ is bounded, then $S_{\chi}$ is (i) nonempty, (ii) compact, and (iii) invariant. Further, (iv) for all $\epsilon>0$ there exists $T \in \tau$ such that $d\left((q(t), x(t)), S_{\chi}\right)<\epsilon$, $t \in \tau$, for all $t \geq T$.

Proof. See [15]. The proofs of (i), (ii), and (iv) are similar to the corresponding result for continuous dynamical systems 1013 .

The conditions of the lemma are sufficient. They can also be shown to be tight: one can construct hybrid automata that violate any one of the conditions of the lemma that accept infinite executions whose $\omega$ limit set is not invariant. The conditions of the lemma are also sufficient to establish continuity of executions with respect to initial conditions, see [15].

LaSalle's Invariance Principle is a useful tool when studying the stability of conventional, continuous dynamical systems. Lemma 1 allows us to extend this tool to hybrid systems.

Theorem 1 (LaSalle's Invariance Principle). Consider a hybrid automaton $H$ that satisfies the conditions of Lemma 11. Assume there exists a compact invariant set $\Omega \subseteq \mathbf{Q} \times \mathbf{X}$ and let $\Omega_{1}=\Omega \cap \mathrm{Out}^{c}$ and $\Omega_{2}=\Omega \cap$ Out. Furthermore, assume there exists a continuous function $V: \Omega \rightarrow \mathbb{R}$, such that

- for all $(q, x) \in \Omega_{1}, V$ is continuously differentiable with respect to $x$ and $L_{f} V(q, x) \leq 0 ;$ and

- for all $(q, x) \in \Omega_{2}, V(\operatorname{Reset}(q, x)) \leq V(q, x)$. 
Define

$$
\begin{aligned}
& S_{1}=\left\{(q, x) \in \Omega_{1}: L_{f} V(q, x)=0\right\} \\
& S_{2}=\left\{(q, x) \in \Omega_{2}: V(\operatorname{Reset}(q, x))=V(q, x)\right\},
\end{aligned}
$$

and let $M$ be the largest invariant subset of $S_{1} \cup S_{2}$. Then, for all $\left(q_{0}, x_{0}\right) \in \Omega$ every execution $(\tau, q, x) \in \mathcal{E}_{H}^{\infty}\left(q_{0}, x_{0}\right)$ approaches $M$ as $t \rightarrow \tau_{\infty}$.

Proof. Consider an arbitrary state $\left(q_{0}, x_{0}\right) \in \Omega$ and let $\chi=(\tau, q, x) \in \mathcal{E}_{H}^{\infty}\left(q_{0}, x_{0}\right)$. Since $\Omega$ is invariant, $(q(t), x(t)) \in \Omega$ for all $t \in \tau$. Since $\Omega$ is compact and $V$ is continuous, $V(q(t), x(t))$ is bounded from below. Moreover, $V(q(t), x(t))$ is a non-increasing function of $t \in \tau$ (recall that $\tau$ is fully ordered), so therefore the limit $c=\lim _{t \rightarrow \tau_{\infty}(\chi)} V(q(t), x(t))$ exists.

Since $\Omega$ is bounded, $x$ is bounded, and therefore the $\omega$ limit set $S_{\chi}$ is nonempty. Moreover, since $\Omega$ is closed, $S_{\chi} \subset \Omega$. By definition, for any $(\hat{q}, \hat{x}) \in$ $S_{\chi}$, there exists a sequence $\left\{\theta_{n}\right\}_{n=0}^{\infty}, \theta_{n} \in \tau$, such that $\theta_{n} \rightarrow \tau_{\infty}$ and $\left(q\left(\theta_{n}\right), x\left(\theta_{n}\right)\right)$ $\rightarrow(\hat{q}, \hat{x})$ as $n \rightarrow \infty$. Then,

$$
V(\hat{q}, \hat{x})=V\left(\lim _{n \rightarrow \infty}\left(q\left(\theta_{n}\right), x\left(\theta_{n}\right)\right)=\lim _{n \rightarrow \infty} V\left(q\left(\theta_{n}\right), x\left(\theta_{n}\right)\right)=c,\right.
$$

by continuity of $V$. Since $S_{\chi}$ is invariant (Lemma 1), it follows that $L_{f} V(\hat{q}, \hat{x})=0$ if $(\hat{q}, \hat{x}) \notin$ Out, and $V(\operatorname{Reset}(\hat{q}, \hat{x}))=V(\hat{q}, \hat{x})$ if $(\hat{q}, \hat{x}) \in$ Out. Therefore, $S_{\chi} \subset$ $S_{1} \cup S_{2}$, which implies that $S_{\chi} \subset M$ since $S_{\chi}$ is invariant. Moreover, by (iv) in Lemma 1, the execution $\chi$ approaches $S_{\chi}$, and hence $M$, as $t \rightarrow \tau_{\infty}$.

\section{Zeno Hybrid Automata}

Zeno hybrid automata accept executions with infinitely many discrete transitions within a finite time interval. Such systems are hard to analysis and simulate in a way that gives constructive information about the behavior of the real system. It is therefore important to be able to determine if a model is Zeno and in applicable cases remove Zenoness. These problems have been discussed in 45]. In this section, some further characterization of Zeno executions are made. Recall that an infinite execution $\chi$ is Zeno if $\tau_{\infty}(\chi)=\sum_{i=0}^{\infty}\left(\tau_{i}^{\prime}-\tau_{i}\right)$ is bounded.

Definition 11 (Zeno Hybrid Automaton). A hybrid automaton $H$ is Zeno if there exists $\left(q_{0}, x_{0}\right) \in$ Init such that all executions in $\mathcal{E}_{H}^{\infty}\left(q_{0}, x_{0}\right)$ are Zeno 4

Example 1. The hybrid automaton in Figure 1 is Zeno. This is easily checked by explicitly deriving the time intervals $\tau_{i}^{\prime}-\tau_{i}$, which in this case gives a converging geometric series. Figure 2 shows an execution accepted by the automaton.

We make the following two straightforward observations.

\footnotetext{
${ }^{4}$ An alternative definition is to say that a hybrid automaton is Zeno if there is at least one Zeno execution in $\mathcal{E}_{H}^{\infty}\left(q_{0}, x_{0}\right)$. In that case, a non-deterministic Zeno hybrid automaton may accept both Zeno and non-Zeno executions, which may be an undesirable feature for instance in Reach set calculations. For deterministic hybrid automata the two definitions coincide.
} 


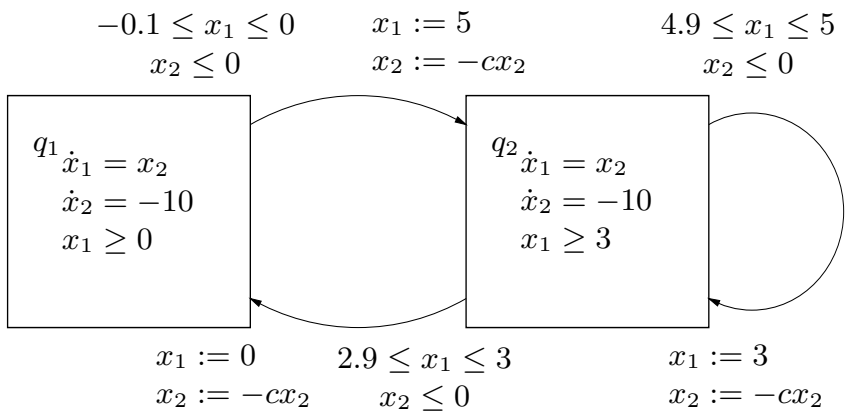

Fig. 1. An example of a Zeno hybrid automaton.

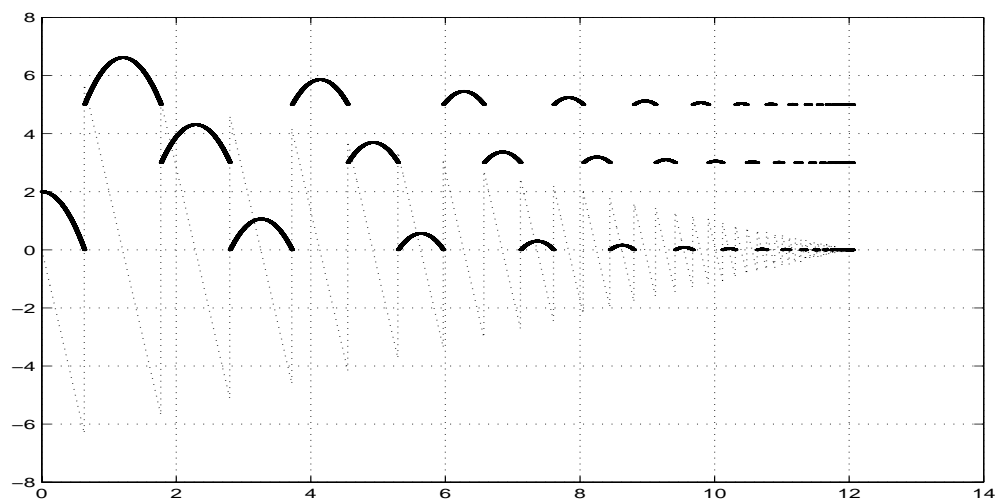

Fig. 2. An example of an execution for the hybrid automaton in Example 1. The continuous part of the state is shown: $x_{1}$ (solid) and $x_{2}$ (dotted).

Proposition 5. A hybrid automaton is Zeno only if the graph $(\mathbf{Q}, E)$ has a cycle.

Proposition 6. If there exists a finite collection of states $\left\{\left(q_{i}, x_{i}\right)\right\}_{i=1}^{N}$ such that

$-\left(q_{1}, x_{1}\right)=\left(q_{N}, x_{N}\right)$;

- $\left(q_{i}, x_{i}\right) \in \operatorname{Reach}_{H}$ for some $i=1, \ldots, N$; and

$-\left(q_{i+1}, x_{i+1}\right)=\operatorname{Reset}\left(q_{i}, x_{i}\right)$ for all $i=1, \ldots, N-1$;

then there exists a Zeno execution.

Zenoness is critically dependent on the reset relation. For example, if in Example 1 the reset maps $x_{2}:=-c x_{2}$ are replaced by $x_{2}:=x_{2} /\left(d x_{2}-1\right)$, where $d=1 / \sqrt{20 x_{1}\left(\tau_{0}\right)}$, then the time intervals $\tau_{i}^{\prime}-\tau_{i}$ decrease as $\{1 / i\}_{i=0}^{\infty}$. This is a diverging series, so the new hybrid automaton is not Zeno.

If the continuous part of the Zeno execution is bounded, then it has an $\omega$ limit point. We introduce the term Zeno state for such a point. 


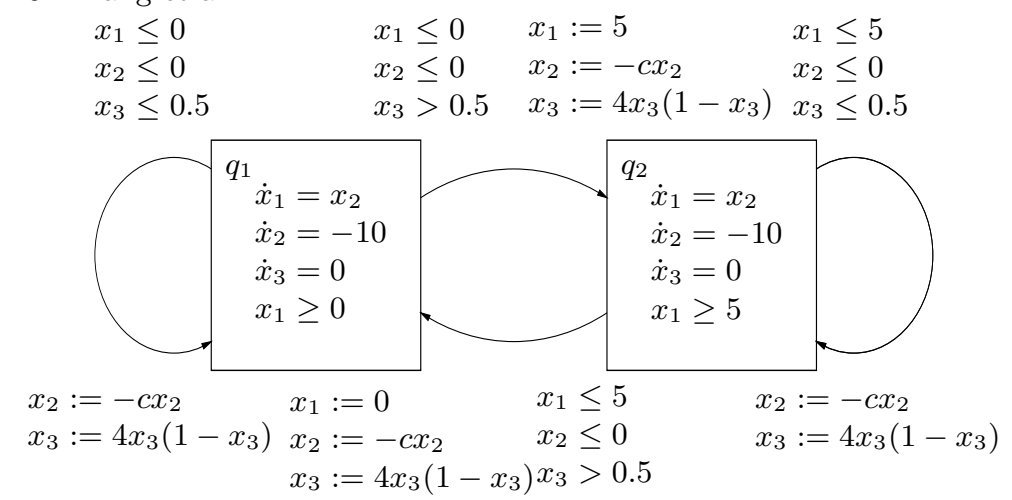

Fig. 3. A hybrid automaton that accepts Zeno executions that do not periodically jump between the discrete states.

Definition 12 (Zeno State). The $\omega$ limit point of a Zeno execution is called the Zeno state.

We use $Z_{\infty} \subset \mathbf{Q} \times \mathbf{X}$ to denote the set of Zeno states, so that $Z_{\infty}$ is the $\omega$ limit set of the Zeno execution. We write $\mathbf{Q}_{\infty}$ for the discrete part of $Z_{\infty}$ and $E_{\infty}$ for the corresponding edges. In Example 1, we have

$$
Z_{\infty}=\left\{\left(q_{1},(0,0)\right),\left(q_{2},(3,0)\right),\left(q_{2},(5,0)\right)\right\}
$$

$\mathbf{Q}_{\infty}=\left\{q_{1}, q_{2}\right\}$, and $E_{\infty}=E$.

It is easy to construct an example with a Zeno executions that do not have a Zeno state. The idea is to let the continuous part of the execution become unbounded as $t \rightarrow \tau_{\infty}(\chi)$. It is also straightforward to derive examples where the set of Zeno states have any number of elements, as well as an infinite but countable or uncountable number of elements. An interesting question is if for a Zeno execution $\chi=(q, x, \tau)$, the discrete part $q$ must become periodic for $t \in \tau$ sufficiently close to $\tau_{\infty}(\chi)$, as in Example 1. The answer is no as illustrated by the following example.

Example 2. Consider the Zeno hybrid automaton in Figure 3 (cf. Example 1). This system does not accept Zeno executions that periodically jump between the two discrete states. A simulation is presented in Figure 4, where $x_{1}$ and $x_{2}$ are shown. The third continuous state is initialized at $x_{3}\left(\tau_{0}\right)=0.9$. The reason for the quasi-periodic behavior is that the reset map of $x_{3}$ is the logistic map and iteration of this map will give any value in $(0,1)$, e.g., [10].

A reset relation Reset is non-expanding, if there exists $\delta \in[0,1]$ such that $\left(q^{\prime}, x^{\prime}\right) \in \operatorname{Reset}(q, x)$ implies $\left\|x^{\prime}\right\| \leq \delta\|x\|$. It is contracting, if there exists $\delta \in$ $[0,1)$ such that $\left(q^{\prime}, x^{\prime}\right) \in \operatorname{Reset}(q, x)$ and $\left(q^{\prime}, y^{\prime}\right) \in \operatorname{Reset}(q, y)$ imply $\left\|x^{\prime}-y^{\prime}\right\| \leq$ $\delta\|y-x\|$. Note that the reset relation has to be a function in the second case. 


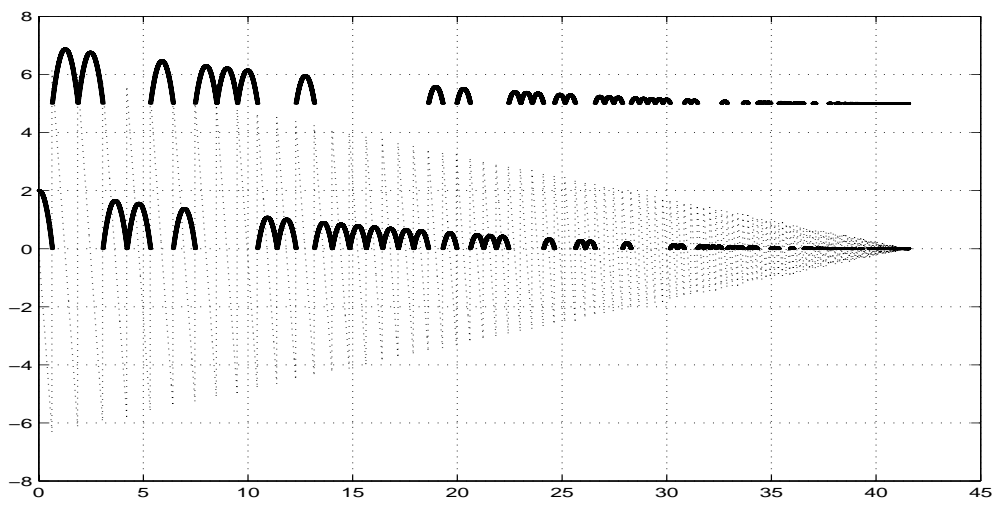

Fig. 4. An example of an execution for the hybrid automaton in Example 2, The continuous states $x_{1}$ (solid) and $x_{2}$ (dotted) are shown. Note how they illustrate the quasi-periodicity.

For smooth dynamical systems, a Lipschitz assumption on the vector field excludes finite escape time. This is not a sufficient condition for hybrid systems. However, if the reset relation is non-expanding (in addition to the Lipschitz assumption on $f(q, \cdot)$ ), then the continuous state is bounded along executions.

Lemma 2. Consider a hybrid automaton with non-expanding reset relation. Then, there exists $c>0$ such that for all executions $\chi=(\tau, q, x) \in \mathcal{E}_{H}$ and $t \in \tau$,

$$
\|x(t)\| \leq\left(\left\|x\left(\tau_{0}\right)\right\|+1\right) e^{c\left(t-\tau_{0}\right)}-1
$$

Proof. The proof, see [15], is similar to the corresponding result for continuous systems 10, Proposition 5.3].

When $x(\cdot)$ is bounded, the Bolzano-Weierstrass Property implies that there exists at least one Zeno state for each Zeno execution. If the continuous part of the reset relation is the identity map, then the continuous part of the Zeno state is a singleton, as proved next.

Theorem 2. Consider a hybrid automaton such that $\left(q^{\prime}, x^{\prime}\right) \in \operatorname{Reset}(q, x)$ implies $x^{\prime}=x$. Then, for every Zeno execution $\chi=(\tau, q, x)$, it holds that $Z_{\infty}=$ $\mathbf{Q}_{\infty} \times\{\hat{x}\}$ for some $\mathbf{Q}_{\infty} \subseteq \mathbf{Q}$ and $\hat{x} \in \mathbf{X}$.

Proof. For all sequences $\left\{\theta_{i}\right\}_{i=0}^{\infty}, \theta_{i} \in \tau$, such that $\theta_{i} \rightarrow \tau_{\infty}$, suppose $\theta_{i} \in$ $\left[\tau_{n_{i}}, \tau_{n_{i}}^{\prime}\right]$, where $n_{i} \rightarrow \infty$ as $i \rightarrow \infty$. We have

$$
\begin{aligned}
x\left(\theta_{i}\right) & =x\left(\tau_{n_{i}}\right)+\int_{\tau_{n_{i}}}^{\theta_{i}} f\left(q\left(\tau_{n_{i}}\right), x(\tau)\right) d \tau \\
& =x\left(\tau_{n_{i}}\right)+\left(\theta_{i}-\tau_{n_{i}}\right) f\left(q\left(\tau_{n_{i}}\right),\left(x_{1}\left(\xi_{n_{i}}^{1}\right), \ldots, x_{n}\left(\xi_{n_{i}}^{n}\right)\right)^{T}\right),
\end{aligned}
$$


for some $\xi_{n_{i}}^{1}, \ldots, \xi_{n_{i}}^{n} \in\left[\tau_{n_{i}}, \tau_{n_{i}}^{\prime}\right]$. Hence, for all $k>\ell \geq 0$,

$$
\begin{aligned}
x\left(\theta_{k}\right)=x & \left(\theta_{\ell}\right)+\left(\tau_{n_{\ell}}^{\prime}-\theta_{\ell}\right) f\left(q\left(\tau_{n_{\ell}}\right),\left(x_{1}\left(\xi_{n_{\ell}}^{1}\right), \ldots, x_{n}\left(\xi_{n_{\ell}}^{n}\right)\right)^{T}\right) \\
& +\sum_{i=n_{\ell}+1}^{n_{k}-1}\left(\tau_{i}^{\prime}-\tau_{i}\right) f\left(q\left(\tau_{i}\right),\left(x_{1}\left(\xi_{i}^{1}\right), \ldots, x_{n}\left(\xi_{i}^{n}\right)\right)^{T}\right) \\
& +\left(\theta_{k}-\tau_{n_{k}}\right) f\left(q\left(\tau_{n_{k}}\right),\left(x_{1}\left(\xi_{n_{k}}^{1}\right), \ldots, x_{n}\left(\xi_{n_{k}}^{n}\right)\right)^{T}\right),
\end{aligned}
$$

which gives that

$$
\left\|x\left(\theta_{k}\right)-x\left(\theta_{\ell}\right)\right\| \leq K \sum_{i=n_{\ell}}^{n_{k}}\left(\tau_{i}^{\prime}-\tau_{i}\right),
$$

where $K>0$ is a constant such that $\|f(q, x)\| \leq K$ for all $(q, x) \in \mathbf{Q} \times \mathbf{X}$. Such constant exists due to Lemma 2. By the fact that $\sum_{i=0}^{\infty}\left(\tau_{i}^{\prime}-\tau_{i}\right)<\infty$, we know that $\left\{x\left(\theta_{i}\right)\right\}_{i=0}^{\infty}$ is a Cauchy sequence. The space $\mathbf{X}=\mathbb{R}^{n}$ is complete, so the sequence has a limit $\hat{x}=\lim _{i \rightarrow \infty} x\left(\theta_{i}\right)$. This limit is independent of the choice of sequence $\left\{\theta_{i}\right\}_{i=0}^{\infty}$, as follows from the following argument. Consider two sequences $\left\{\alpha_{i}\right\}_{i=0}^{\infty}$ and $\left\{\beta_{i}\right\}_{i=0}^{\infty}, \alpha_{i}, \beta_{i} \in \tau$, such that $\alpha_{i} \rightarrow \tau_{\infty}$ and $\beta_{i} \rightarrow \tau_{\infty}$. Suppose $\alpha_{i} \in\left[\tau_{m_{i}}, \tau_{m_{i}}^{\prime}\right]$ and $\beta_{i} \in\left[\tau_{n_{i}}, \tau_{n_{i}}^{\prime}\right]$, where $m_{i} \rightarrow \infty$ and $n_{i} \rightarrow \infty$ as $i \rightarrow \infty$, and $m_{i} \geq n_{i}$. Then,

$$
\begin{aligned}
x\left(\alpha_{i}\right)=x & \left(\beta_{i}\right)+\left(\tau_{n_{i}}^{\prime}-\beta_{i}\right) f\left(q\left(\tau_{n_{i}}\right),\left(x_{1}\left(\xi_{n_{i}}^{1}\right), \ldots, x_{n}\left(\xi_{n_{i}}^{n}\right)\right)^{T}\right) \\
& +\sum_{j=n_{i}+1}^{m_{i}-1}\left(\tau_{j}^{\prime}-\tau_{j}\right) f\left(q\left(\tau_{j}\right),\left(x_{1}\left(\xi_{j}^{1}\right), \ldots, x_{n}\left(\xi_{j}^{n}\right)\right)^{T}\right) \\
& +\left(\alpha_{i}-\tau_{m_{i}}\right) f\left(q\left(\tau_{m_{i}}\right),\left(x_{1}\left(\xi_{m_{i}}^{1}\right), \ldots, x_{n}\left(\xi_{m_{i}}^{n}\right)\right)^{T}\right) .
\end{aligned}
$$

This gives that $\left\|x\left(\alpha_{i}\right)-x\left(\beta_{i}\right)\right\| \leq K \sum_{j=n_{i}}^{m_{i}}\left(\tau_{j}^{\prime}-\tau_{j}\right)$. Hence, $\left\|x\left(\alpha_{i}\right)-x\left(\beta_{i}\right)\right\| \rightarrow 0$ as $i \rightarrow \infty$, which shows that both sequences have the same limit. This completes the proof.

Note that Theorem 2 gives the structure of the Zeno state for the large class of hybrid systems called switched systems [9], since these systems can be modeled as hybrid automata with identity reset relation.

If the reset relation is contracting and $\left(q^{\prime}, x^{\prime}\right) \in \operatorname{Reset}(q, 0)$ implies that $x^{\prime}$ is the origin, then the continuous part of the Zeno state is also the origin.

Theorem 3. Consider a Zeno hybrid automaton with contracting reset relation and such that $\left(q^{\prime}, x^{\prime}\right) \in \operatorname{Reset}(q, 0)$ implies $x^{\prime}=0$. Then, for every Zeno execution $\chi=(\tau, q, x)$, it holds that $Z_{\infty}=\mathbf{Q}_{\infty} \times\{0\}$ for some $\mathbf{Q}_{\infty} \subseteq \mathbf{Q}$.

Proof. For all sequences $\left\{\theta_{i}\right\}_{i=0}^{\infty}, \theta_{i} \in \tau$, such that $\theta_{i} \rightarrow \tau_{\infty}$, suppose $\theta_{i} \in$ $\left[\tau_{n_{i}}, \tau_{n_{i}}^{\prime}\right]$, where $n_{i} \rightarrow \infty$ as $i \rightarrow \infty$. We have

$$
\begin{aligned}
\left\|x\left(\theta_{i}\right)\right\| & \leq\left\|x\left(\tau_{n_{i}}\right)\right\|+\left\|\int_{\tau_{n_{i}}}^{\theta_{i}} f\left(q\left(\tau_{n_{i}}\right), x(\tau)\right) d \tau\right\| \\
& \leq\left\|x\left(\tau_{n_{i}}\right)\right\|+K\left(\tau_{n_{i}}^{\prime}-\tau_{n_{i}}\right),
\end{aligned}
$$


where $K>0$ is the same constant as in the proof of Theorem 2 Using the fact that $\left\|x\left(\tau_{n_{i}}\right)\right\| \leq \delta\left\|x\left(\tau_{n_{i}-1}^{\prime}\right)\right\|$, it follows that

$$
\begin{aligned}
\left\|x\left(\theta_{i}\right)\right\| & \leq \delta\left\|x\left(\tau_{n_{i}-1}^{\prime}\right)\right\|+K\left(\tau_{n_{i}}^{\prime}-\tau_{n_{i}}\right) \\
& =\delta\left\|x\left(\tau_{n_{i}-1}\right)+\int_{\tau_{n_{i}-1}}^{\tau_{n_{i}-1}^{\prime}} f\left(q\left(\tau_{n_{i}-1}\right), x(\tau)\right) d \tau\right\|+K\left(\tau_{n_{i}}^{\prime}-\tau_{n_{i}}\right) \\
& \leq \delta\left\|x\left(\tau_{n_{i}-1}\right)\right\|+K \delta\left(\tau_{n_{i}-1}^{\prime}-\tau_{n_{i}-1}\right)+K\left(\tau_{n_{i}}^{\prime}-\tau_{n_{i}}\right) .
\end{aligned}
$$

By induction,

$$
\left\|x\left(\theta_{i}\right)\right\| \leq \delta^{n_{i}}\left\|x\left(\tau_{0}\right)\right\|+K \sum_{m=0}^{n_{i}} \delta^{n_{i}-m}\left(\tau_{m}^{\prime}-\tau_{m}\right) .
$$

Since

$$
\sum_{n_{i}=0}^{\infty} K \sum_{m=0}^{n_{i}} \delta^{n_{i}-m}\left(\tau_{m}^{\prime}-\tau_{m}\right)=K \sum_{m=0}^{\infty}\left(\tau_{m}^{\prime}-\tau_{m}\right) \sum_{n_{i}=0}^{\infty} \delta^{n_{i}}=\frac{K \tau_{\infty}}{1-\delta}<\infty
$$

it holds that $K \sum_{m=0}^{n_{i}} \delta^{n_{i}-m}\left(\tau_{m}^{\prime}-\tau_{m}\right) \rightarrow 0$ as $n_{i} \rightarrow \infty$. This yields that $\left\|x\left(\theta_{i}\right)\right\| \rightarrow 0$ as $i \rightarrow \infty$, which, hence, completes the proof.

A generalization of Theorem 3 holds if we change the assumption to that $\left(q^{\prime}, x^{\prime}\right) \in$ Reset $\left(q, x^{*}\right)$ implies $x^{\prime}=x^{*}$ for some $x^{*} \in$ Dom, see [15].

For a large class of Zeno hybrid automata, the continuous part of the Zeno state is located on the intersection of the boundaries of $\operatorname{Dom}(q, \cdot)$ for $q \in \mathbf{Q}_{\infty}$. Next this result is stated for hybrid automata with non-expanding reset relation. Recall that $I(q)=\{x \in \mathbf{X}:(q, x) \in \operatorname{Dom}\}$.

Proposition 7. Consider a hybrid automaton $H$ with non-expanding reset relation. Assume it accepts a Zeno execution $\chi=(\tau, q, x) \in \mathcal{E}_{H}^{\infty}$ with set of Zeno states $Z_{\infty}=\left\{\left(q_{i}, x_{i}\right\}_{i=1}^{N}, N \geq 1\right.$. If, for all $i \in\{1, \ldots, N\}$ and $x \in I\left(q_{i}\right)^{o}$, $\operatorname{Reset}\left(q_{i}, x\right)=\emptyset$, then $x_{i} \in \partial I\left(q_{i}\right)$ for all $i \in\{1, \ldots, N\}$. Furthermore, if there exists $\hat{x} \in$ Dom such that for all $i \in\{1, \ldots, N\}, x_{i}=\hat{x}$, then $\hat{x} \in \bigcap_{i=1}^{N} \partial I\left(q_{i}\right)$.

Proof. See [15].

It follows from Proposition 7 that if the boundaries of $I(\cdot)$ are not intersecting, then there exist no Zeno executions with non-empty Zeno state and $N>1$. Proposition [7 is thus a refinement of the condition given in Proposition [5] which states that a hybrid automaton is non-Zeno if the graph $(\mathbf{Q}, E)$ has no cycle.

\section{Conclusions}

Motivated by numerous assumptions like "In this paper, we assume that the switched system is live and nonZeno" 3] and suggestions like "Additional work is needed in determining the role that Zeno-type control might play in hybrid 
system supervision" [6], we have extended some classical results to hybrid systems, using tools that capture both non-Zeno and Zeno executions. We have also tried to illustrate some of the nature of Zeno by characterizing Zeno executions and Zeno states for a few quite broad classes of hybrid systems. Zeno hybrid automata are characterized from a geometric point of view in [1].

\section{Acknowledgments}

The authors would like to thank Magnus Egerstedt and Slobodan Simić for helpful discussions.

\section{References}

1. M. Branicky. Multiple Lyapunov functions and other analysis tools for switched and hybrid systems. IEEE Transactions on Automatic Control, 43(4):475-482, April 1998.

2. M. Branicky, E. Dolginova, and N. Lynch. A toolbox for proving and maintaining hybrid specifications. In P. Antsaklis, W. Kohn, A. Nerode, and S. Sastry, editors, Hybrid Systems IV, number 1273 in LNCS, pages 18-30. Springer Verlag, 1997.

3. K. X. He and M. D. Lemmon. Lyapunov stability of continuous-valued systems under the supervision of discrete-event transition systems. In Hybrid Systems: Computation and Control, volume 1386 of Lecture Notes in Computer Science. Springer-Verlag, Berlin, 1998.

4. K. H. Johansson, M. Egerstedt, J. Lygeros, and S. Sastry. On the regularization of Zeno hybrid automata. Systems $\&$ Control Letters, 38:141-150, 1999.

5. K. H. Johansson, J. Lygeros, S. Sastry, and M. Egerstedt. Simulation of Zeno hybrid automata. In IEEE Conference on Decision and Control, Phoenix, AZ, 1999.

6. M. D. Lemmon, K. X. He, and I Markovsky. Supervisory hybrid systems. IEEE Control Systems Magazine, 19(4):42-55, 1999.

7. J. Lygeros, K. H. Johansson, S. Sastry, and M. Egerstedt. On the existence of executions of hybrid automata. In IEEE Conference on Decision and Control, Phoenix, AZ, 1999.

8. J. Lygeros, C. Tomlin, and S. Sastry. Controllers for reachability specifications for hybrid systems. Automatica, 35(3), March 1999.

9. A. S. Morse. Control using logic-based switching. In Alberto Isidori, editor, Trends in Control. A European Perspective, pages 69-113. Springer, 1995.

10. S. Sastry. Nonlinear Systems: Analysis, Stability, and Control. Springer-Verlag, New York, 1999.

11. S Simić, K H Johansson, S Sastry, and J Lygeros. Towards a geometric theory of hybrid systems. In Hybrid Systems: Computation and Control, Pittsburgh, PA, 2000.

12. A. J. van der Schaft and J. M. Schumacher. Complementarity modeling of hybrid systems. IEEE Transactions on Automatic Control, 43(4):483-490, April 1998.

13. S. Wiggins. Introduction to Applied Nonlinear Dynamical Systems and Chaos. Springer-Verlag, New York, 1990.

14. H. Ye, A. Michel, and L. Hou. Stability theory for hybrid dynamical systems. IEEE Transactions on Automatic Control, 43(4):461-474, April 1998.

15. J. Zhang. Dynamical systems revisited: Hybrid systems with Zeno executions. Master's thesis, Dept of EECS, University of California, Berkeley, 1999. 